\title{
Background Studies for the MINER Coherent Neutrino Scattering Reactor Experiment
}

G. Agnolet ${ }^{\mathrm{a}}$, W. Baker ${ }^{\mathrm{a}}$, D. Barker ${ }^{\mathrm{b}}$, R. Beck ${ }^{\mathrm{a}}$, T.J. Carroll ${ }^{\mathrm{c}}$, J. Cesar ${ }^{\mathrm{c}}$, P. Cushman ${ }^{\mathrm{b}}$, J.B. Dent ${ }^{\mathrm{d}}$, S. De Rijck ${ }^{\mathrm{c}}$, B. Dutta ${ }^{\mathrm{a}}$, W. Flanagan ${ }^{\mathrm{c}}$, M. Fritts ${ }^{\mathrm{b}}$, Y. Gao ${ }^{\mathrm{a}, \mathrm{e}}$, H.R. Harris ${ }^{\mathrm{a}}$, C.C. Hays ${ }^{\mathrm{a}}$, V. Iyer ${ }^{\mathrm{f}}$, A. Jastram ${ }^{\mathrm{a}}$, F. Kadribasic ${ }^{\mathrm{a}}$, A. Kennedy ${ }^{\mathrm{b}}$, A. Kubik ${ }^{\mathrm{a}}, \mathrm{K}$. Lang ${ }^{\mathrm{c}}$, R. Mahapatra ${ }^{\mathrm{a}}$, V. Mandic ${ }^{\mathrm{b}}$, C. Marianno ${ }^{g}$, R.D. Martin ${ }^{\mathrm{i}}$, N. Mast ${ }^{\mathrm{b}}$, S. McDeavitt ${ }^{\mathrm{j}}$, N. Mirabolfathi ${ }^{\mathrm{a}}$, B. Mohanty ${ }^{\mathrm{f}}$, K. Nakajima ${ }^{\mathrm{k}}$, J. Newhouse , J.L. Newstead ${ }^{\mathrm{k}}$, I. Ogawa ${ }^{\mathrm{h}}$, D. Phan ${ }^{\mathrm{c}}$, M. Proga ${ }^{\mathrm{c}}$, A. Rajput ${ }^{\mathrm{c}}$, A. Roberts ${ }^{\mathrm{l}}$, G. Rogachev ${ }^{\mathrm{m}}$, R. Salazarc, J. Sander ${ }^{\mathrm{l}}$, K. Senapati ${ }^{\mathrm{f}}$, M. Shimadah ${ }^{\mathrm{h}}$, B. Soubasis ${ }^{\mathrm{c}}$, L. Strigari ${ }^{\mathrm{a}}$, Y. Tamagawa ${ }^{\mathrm{h}}$, W. Teizer ${ }^{\mathrm{a}}$, J.I.C. Vermaak ${ }^{\mathrm{j}}$, A.N. Villano ${ }^{\mathrm{b}}$, J. Walker ${ }^{\mathrm{n}}$, B. Webb ${ }^{\mathrm{a}}$, Z. Wetzel ${ }^{\mathrm{a}}$, S.A. Yadavalli ${ }^{\mathrm{c}}$

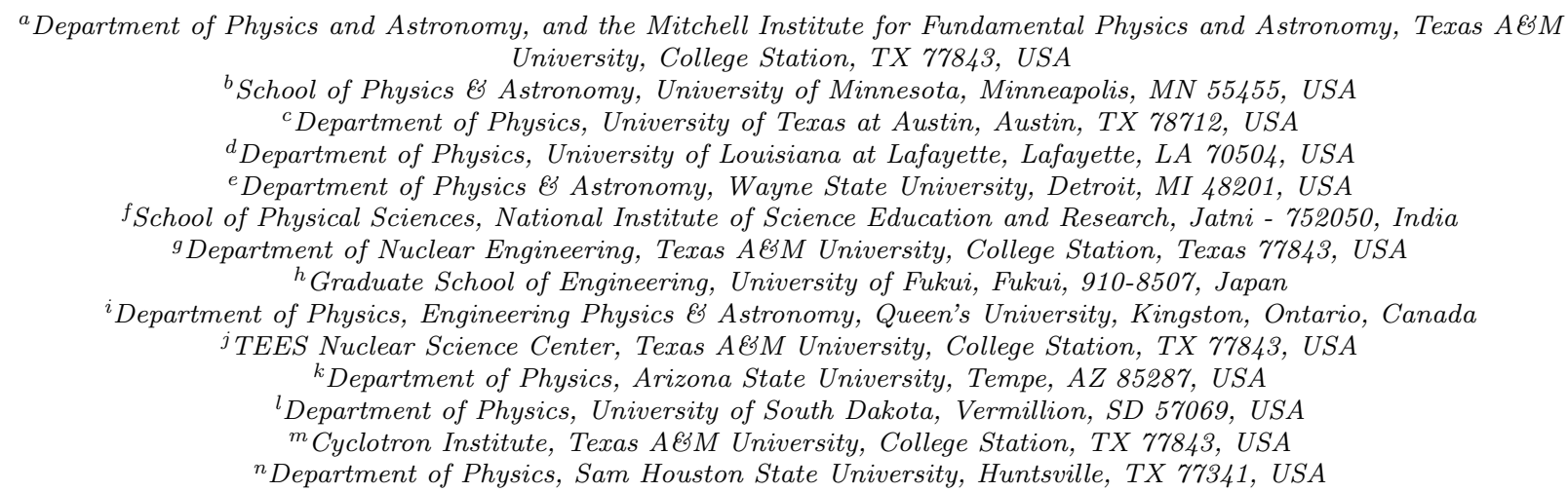

\begin{abstract}
The proposed Mitchell Institute Neutrino Experiment at Reactor (MINER) experiment at the Nuclear Science Center at Texas A\&M University will search for coherent elastic neutrino-nucleus scattering within close proximity (about 2 meters) of a $1 \mathrm{MW}$ TRIGA nuclear reactor core using low threshold, cryogenic germanium and silicon detectors. Given the Standard Model cross section of the scattering process and the proposed experimental proximity to the reactor, as many as 5 to 20 events $/ \mathrm{kg} /$ day are expected. We discuss the status of preliminary measurements to characterize the main backgrounds for the proposed experiment. Both in situ measurements at the experimental site and simulations using the MCNP and GEANT4 codes are described. A strategy for monitoring backgrounds during data taking is briefly discussed.
\end{abstract}

\section{Introduction}

The cross section for the coherent elastic scattering of neutrinos off of nuclei (CE $\nu$ NS) 1 is a longstanding prediction of the Standard Model, but has yet to be measured experimentally in part due to the extremely low energy threshold needed for detection with typical high flux neutrino sources such as nuclear reactors. Improvements in semiconductor detector technologies [2] which utilize the Neganov-Luke phonon amplification method 3 have brought $\mathrm{CE} \nu \mathrm{NS}$ detection within reach. The Mitchell Institute Neutrino Experiment at Reactor (MINER) experiment, currently under development at the Nuclear Science Center (NSC) at Texas A\&M University, will leverage this detector technology to detect CE $\nu$ NS and measure its cross section. If successful, the $\mathrm{CE} \nu \mathrm{NS}$ interactions can be used to probe new physics scenarios including a search for sterile neutrino oscillations, the neutrino magnetic moment, and other processes beyond the Standard Model 44 7]. The experiment will utilize a megawatt-class TRIGA (Training, Research, Isotopes, General Atomics) pool reactor stocked with low-enriched (about $20 \%{ }^{235} \mathrm{U}$. This facility has the unique 
advantage of possessing a movable core and provides access to deploy detectors as close as about $1 \mathrm{~m}$ from the reactor, allowing for a varying distance from the neutrino source to the detector. At these short baselines, we expect to detect as many as 20 events $/ \mathrm{kg} /$ day in the range of recoil energy between 10 and $1000 \mathrm{eV}$ nr. This estimate is obtained by integrating the Standard Model differential cross-section over the neutrino energy spectrum of a megawatt ${ }^{235} \mathrm{U}$ reactor and integrating the nuclear recoil energy from the specified sensitivity threshold up to the kinematic cutoff, as described in 44, 7]. The rate is cut to approximately a third if the detection threshold is instead $100 \mathrm{eV}_{\mathrm{nr}}$, and diminishes as $r^{-2}$ with increasing distance, e.g. to as many as 5 events $/ \mathrm{kg} /$ day at $2 \mathrm{~m}$.

An important aspect of the proposed experiment are the backgrounds induced by both the core and environmental sources. These backgrounds include gammas and neutrons from the reactor, muons and muon-induced neutrons from cosmic rays, and ambient gammas. The rate of such backgrounds must be comparable to or below the expected rate of the neutrino recoil signal. We take a rate of 100 events $/ \mathrm{kg} / \mathrm{day}$ in the range of recoil energy between 10 and $1000 \mathrm{eV}_{\mathrm{nr}}$ as the target level of acceptable background rate, corresponding to a signal to background ratio of about 0.05 to 0.2 . It has been demonstrated in [8] that a signal rate of 10 over a background rate of 100 events $/ \mathrm{kg} /$ day is discoverable at the $5 \sigma$ level after a few months of integrated run time using a binned profile likelihood test statistic with marginalization over the background and flux normalization and assuming $2 \%$ systematic uncertainty. Events outside of this 10 to $1000 \mathrm{eV}_{\mathrm{nr}}$ energy window are acceptable to a level of about $100 \mathrm{~Hz}$ total event rate, dictated by the sampling rate of the data acquisition system. These higher energy events can serve to normalize backgrounds in the lower energy signal region.

The paper is organized as follows. In Section 2, a brief description of the experimental location is given. Section 3 describes the modeling of the reactor core and experiment in the MCNP and GEANT4 framework. Sections 4. 5, and 6 describe the in situ measurements of the gamma, neutron, and cosmic muon backgrounds respectively, including comparison to the simulation for the gamma and neutron backgrounds. Section 7 combines the simulation with the in situ measurements to estimate a background rate in the detectors given a preliminary shielding design. Finally, status and prospects are described in Section 8.

\section{Description of Experimental Site}

The NSC reactor facility pool is surrounded by roughly 2 meters of high density concrete (about $3.5 \mathrm{~g} / \mathrm{cm}^{3}$ density) which acts as a shield to the high flux of neutron and gamma byproducts in the reactor. A cavity in this wall, dubbed the "Thermal Column", was used in the past to facilitate close proximity to the reactor for material neutron irradiation. The cavity is located in the lower research area of the NSC and is in the same horizontal plane as the reactor core (see Figure 1). The cavity has many advantages as an experimental location, including the ability to access an area in very close proximity to the core, a natural overburden provided by the concrete wall to reduce the rate of cosmic muons, and an open area to allow placement of optimized shielding between the core and the detectors. A schematic diagram of the Thermal Column can be seen in Figure 1 and a photograph from the outside of the cavity is shown in Figure 2.

\section{Background Simulation}

\subsection{Reactor Core Model}

Fission processes in the reactor produce large fluxes of both gammas and neutrons near the core. The energy spectrum and production rate of these backgrounds are predicted using a core model developed at the NSC, shown in Figure 3, and applied in the MCNP 9 framework. The TRIGA reactor of the NSC features a 90 fuel element, low-enriched uranium core operating at a nominal power of $1 \mathrm{MW}$. The fuel burn-up of the relatively new core (installed in 2006) is modeled in a 15 axial layer configuration for each fuel element and includes a wide range of fission products in the fuel material resulting in a high detail model of the reactor.

Using the MCNP reactor core model, we calculated the neutron energy spectrum produced by the reactor shown on the left in Figure 4 , with fluxes of $5.8 \times 10^{11} \mathrm{~cm}^{-2} \mathrm{~s}^{-1}$ fast component (> $100 \mathrm{keV}$ kinetic energy) 


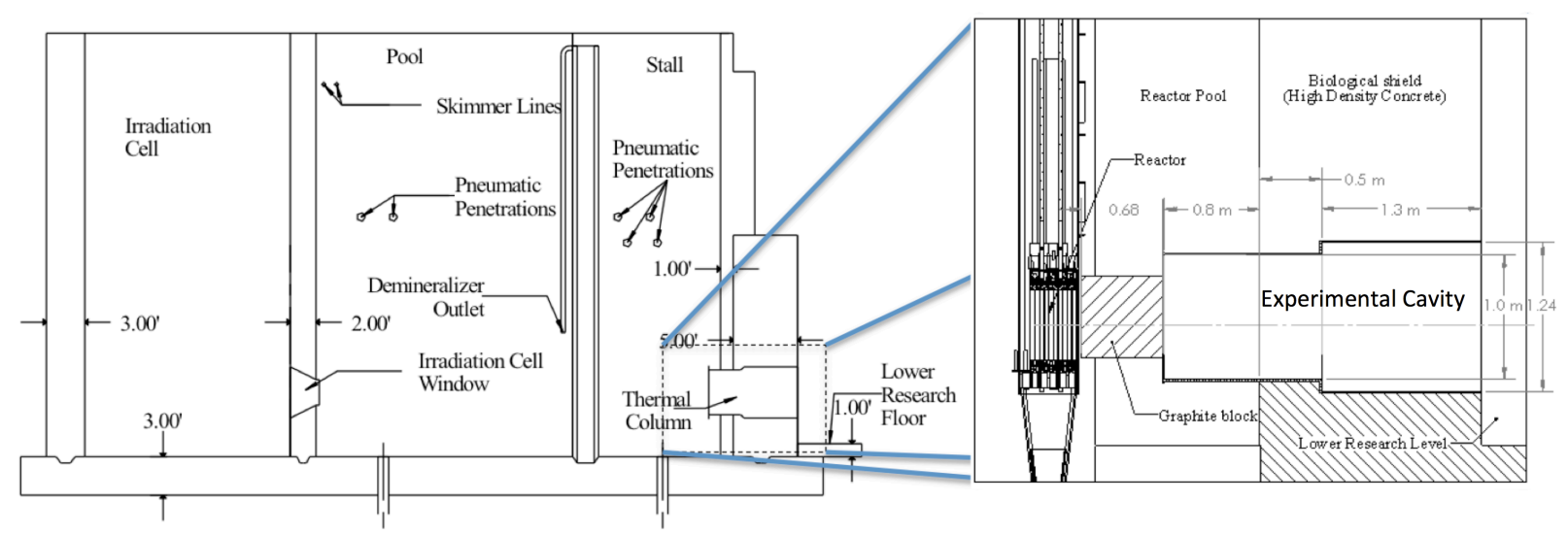

Figure 1: Schematic side view of the reactor pool and experimental cavity where the proposed detector and shielding will be constructed.

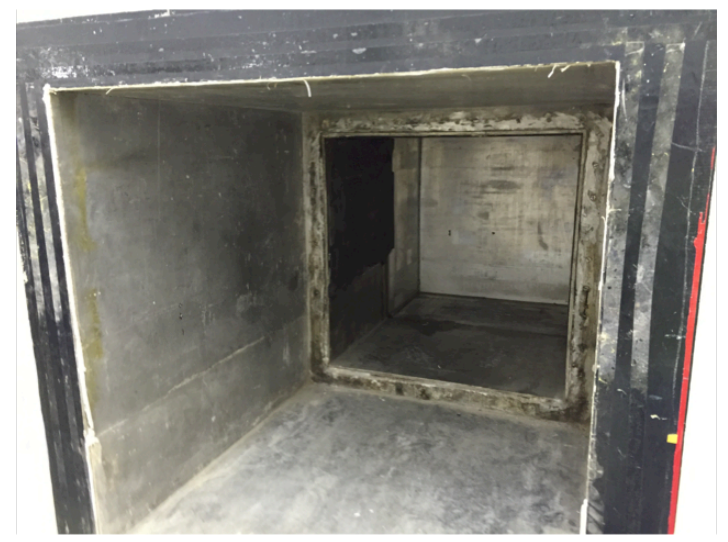

Figure 2: Photograph from the outside of the empty experimental cavity.

and $7.7 \times 10^{12} \mathrm{~cm}^{-2} \mathrm{~s}^{-1}$ thermal component $(<0.625 \mathrm{eV}$ kinetic energy). A moderator can be used to convert the fast neutron flux to a thermal flux which can be shielded using a thermal neutron absorber such as boron, cadmium or gadolinium.

The simulated gamma spectrum is shown on the right in Figure 4 , with a total flux of $9.0 \times 10^{11} \mathrm{~cm}^{-2} \mathrm{~s}^{-1}$. This gamma energy spectrum can be attenuated by conventional high density materials such as lead.

\subsection{GEANT4 Geometry Model}

A model geometry of the experimental hall was constructed in the GEANT4 [10] (v10.2.1) framework. Correct description of the atomic composition of the surrounding materials is critical since backgrounds strongly depend on the materials used and secondary production of backgrounds in these materials must be included. Detailed material descriptions with isotope composition to the level of ppm are provided by the NSC and are included in the GEANT4 model.

Reactor gammas and neutrons were generated with an energy spectrum as produced by the MCNP core model described above. The flux is modeled to originate from a $30 \times 30 \mathrm{~cm}^{2}$ square plane representing the active face of the reactor closest to the experimental cavity. To model different core positions, this source surface was moved to the corresponding core face position. To significantly save computational time, particles were simulated with only momenta along a direction from the reactor core to the experimental cavity, perpendicular to the source plane. The "Shielding" [11] physics list was used in all GEANT4 simulations described in this paper. 

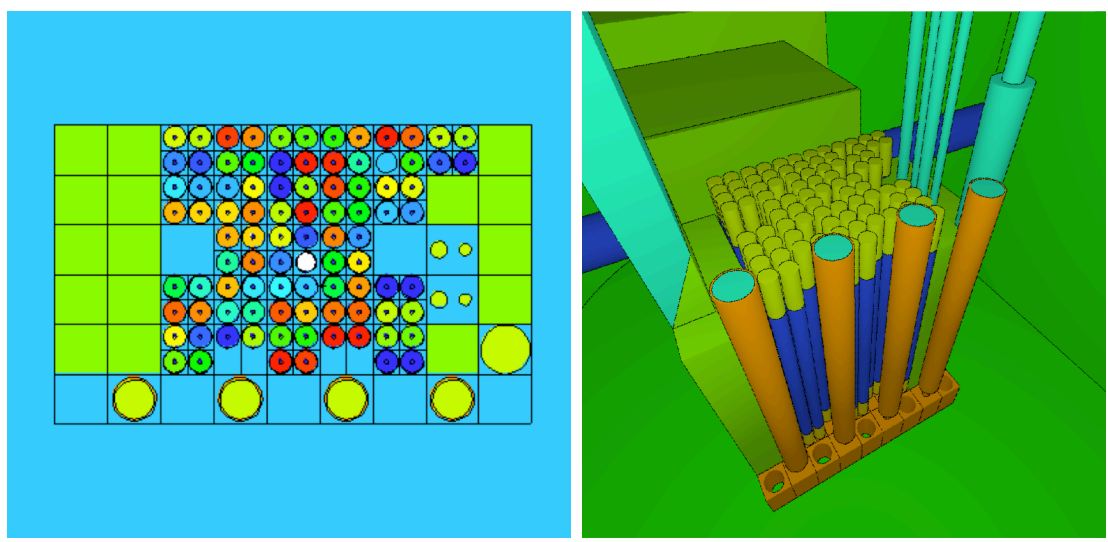

Figure 3: Visualization of the TRIGA reactor core as modeled in MCNP. The small circles in the picture represent the various fuel and control rods while the green rectangles are graphite reflectors. The fuel rods are about $3.58 \mathrm{~cm}$ in diameter and $38.1 \mathrm{~cm}$ in length.
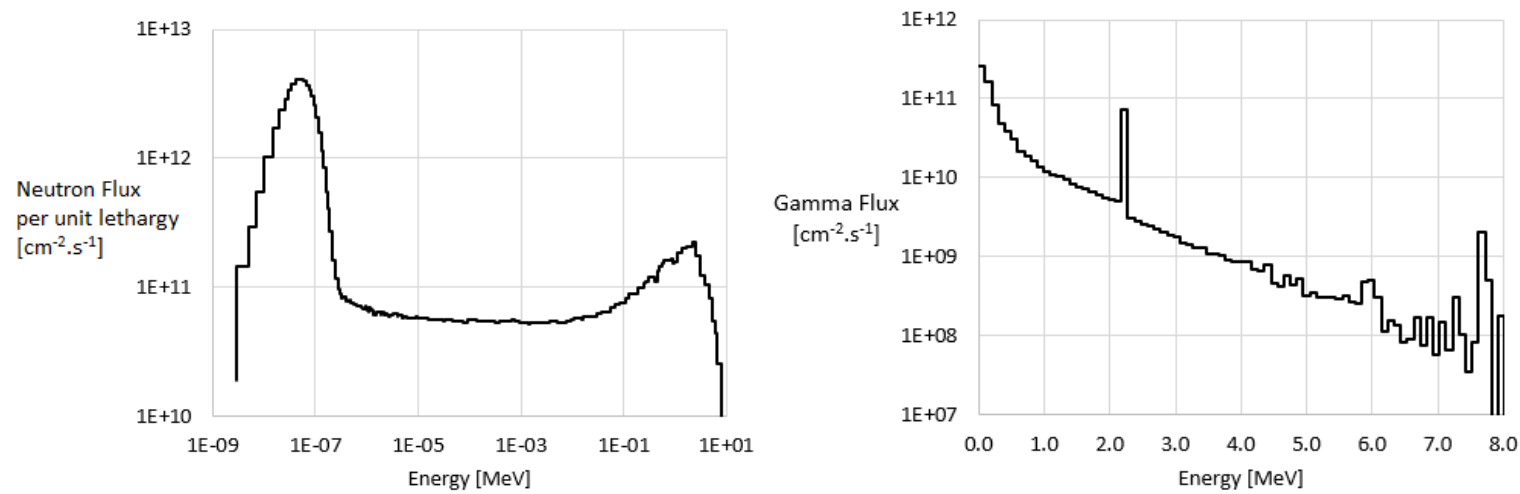

Figure 4: Calculated neutron (left) and gamma (right) spectra just outside the core volume (before shielding), obtained using the MCNP core model as described in the text. The neutron spectrum is bin-by-bin normalized to unit lethargy, with normalization factor given as $E_{A v e} /\left(E_{2}-E_{1}\right)$ where $E_{1}$ and $E_{2}$ are the energy values at the respective bin edges and $E_{A v e}$ is the average of these bin edge values. The $2.2 \mathrm{MeV}$ line in the gamma spectrum is the result of neutron capture on Hydrogen in the surrounding water, while excesses in bins near 6 and $8 \mathrm{MeV}$ are due to statistical fluctuations. The total fluxes from these calculations are $5.8 \times 10^{11} \mathrm{~cm}^{-2} \mathrm{~s}^{-1}$ fast neutrons component $\left(>100 \mathrm{keV}\right.$ kinetic energy), $7.7 \times 10^{12} \mathrm{~cm}^{-2} \mathrm{~s}^{-1}$ thermal neutrons $\left(<0.625 \mathrm{eV}\right.$ kinetic energy), and $9.0 \times 10^{11} \mathrm{~cm}^{-2} \mathrm{~s}^{-1}$ gammas.

Since the initial flux from the reactor is large and the desired target rate at the detectors must be low, we implemented a variance reduction scheme to obtain good statistical significance of the background characteristics at the detector site. GEANT4 has multiple built-in variance reduction schemes available to users, and we chose the 'importance sampling' scheme for this application. In the importance sampling scheme, the geometry is divided up into different regions, each with an importance score assigned. As a particle is propagated across the boundary of two such regions, the ratio of the importance score in the new region over that of the previous region is taken. If this ratio is larger than one, the particle is duplicated a number of times equal to this ratio decreased by one. If less than one, a "Russian Roulette" algorithm is used to determine whether to terminate the particle with a probability equal to the ratio. Particles are then weighted by the inverse of the importance ratio. By increasing the importance value assigned to regions deeper within the shielding, the number of particles making it through to the detector is greatly enhanced. We used 16 to 22 importance regions (depending on distance of the source to the detectors) of equal thickness with importance score increasing by factors of 2 . Large statistics GEANT4 simulations (typically around $10^{9}$ primary particles generated) were run on both the Brazos Computing Cluster at Texas A\&M University 
as well as the Texas Advanced Computing Center (TACC) cluster at the University of Texas at Austin.

A preliminary shielding design was then added to the GEANT4 geometry model to assess the background expected in the full experimental setup. Materials included $1.38 \mathrm{~m}$ of high density borated (5\%) polyethylene as neutron shielding and $30.5 \mathrm{~cm}$ of lead as gamma shielding. Additional lead and polyethylene were included after the initial shielding to reduce backgrounds from secondary particles which include neutrons from $(\gamma, \mathrm{n})$ reactions and gammas released from neutron captures in the shielding materials. This shielding design is shown in Figure 5 . The thickness of shielding materials in this design was chosen based on initial estimates made with simple GEANT4 geometry models but has not yet been optimized.

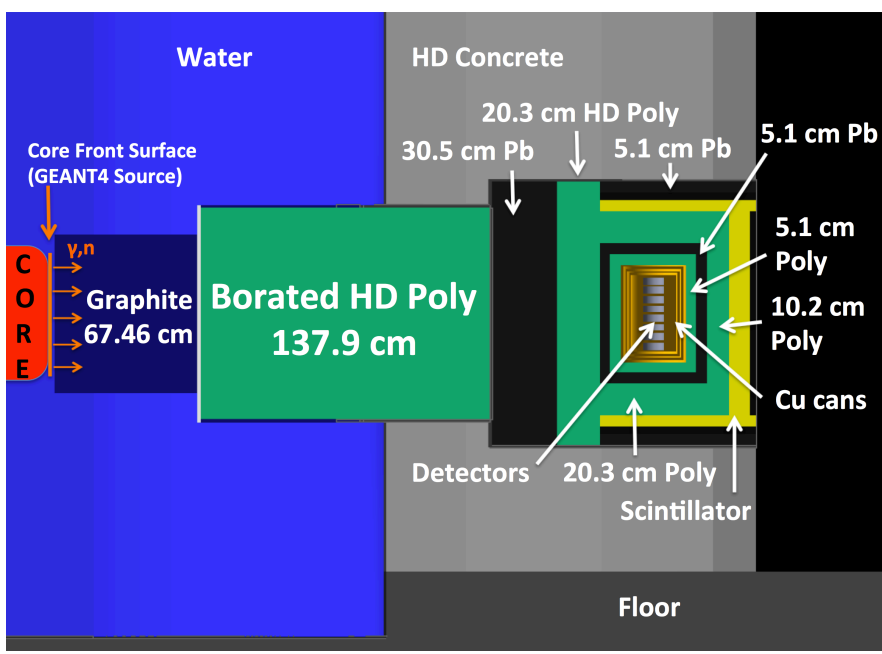

Figure 5: Cross section of the GEANT4 geometry model of the experimental cavity with a preliminary shield design. In this figure, different materials are represented by different colors. Blue: water, Dark Blue: graphite, Green: $5 \%$ Borated polyethylene, Light Gray: HD concrete, Dark Gray: Lead, Gold: Copper, Yellow: Plastic Scintillator. The small disks within the copper are germanium and silicon detectors.

\section{Gamma Background Measurements}

Background measurements have been conducted in the experimental cavity using a commercial High Purity Germanium (HPGe) detector shown in Figure 6 (Canberra GC2020, approx. 0.5 kg). Due to the large volume of water between the detector and the reactor core, these measurements were dominated by gamma interactions. A commercially available shield was used to limit the rate registered by the detector, while maintaining a simple geometry for matching with simulations. The shield is cylindrical, comprised of a 4" layer of low activity lead enclosed externally by a $1 / 2$ " thick layer of steel. Inside the shield cavity, the lead is lined with layers of high purity tin and OFHC copper, approximately 0.04 " and 0.06 " thick, respectively, to block lead X-rays. Measurements were made at reactor powers of $0 \mathrm{~kW}$ (core off), $1 \mathrm{~kW}, 98 \mathrm{~kW}$, and $500 \mathrm{~kW}$ at distances (measured from the face of the core to the face of the experimental cavity) of $3.83 \mathrm{~m}$, $3.33 \mathrm{~m}$, and $2.83 \mathrm{~m}$ (see Figure 7). All measurements consist of 300 seconds of live time. The HPGe detector was calibrated before measurements using a ${ }^{22} \mathrm{Na}$ source, which provides gammas with energy $511 \mathrm{keV}$ and $1274 \mathrm{keV}$.

Measured energy spectra are due to gammas produced in the reactor core and gammas from other sources (e.g., activated materials in the area). To properly compare simulations of the reactor core flux with measurements, the flux that is not coming directly from the reactor core must be subtracted. To perform this subtraction, we use the energy spectra measured at a given core position, while the reactor is turned off. An example of this subtraction is shown in Figure 8. It can be seen that after this subtraction, the only remaining spectral line is the $511 \mathrm{keV}$ electron-positron annihilation line. Other lines present in the initial 


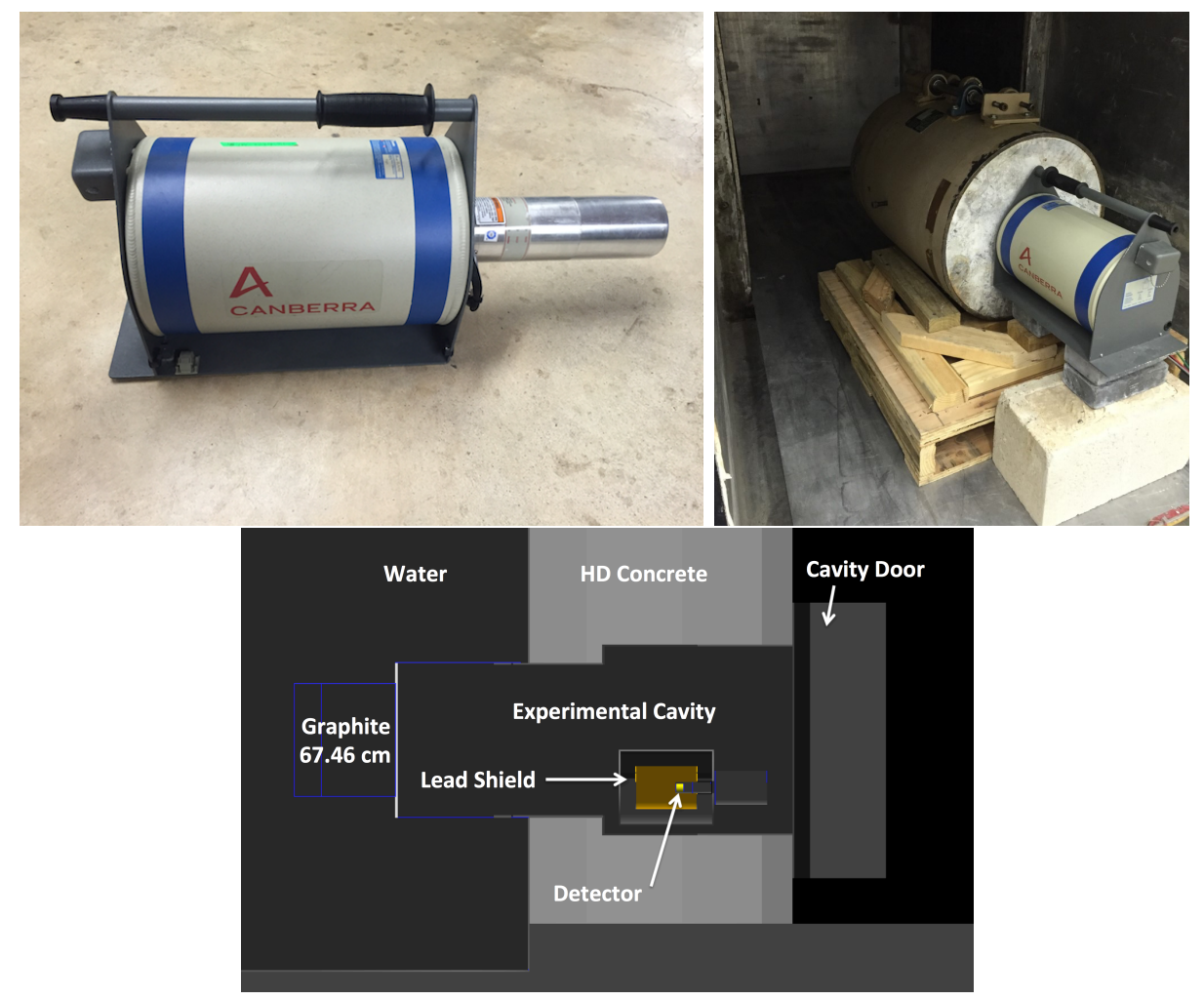

Figure 6: Top Left: Photo of Canberra HPGe detector used in the gamma measurement. Top Right: Placement of HPGe detector and shielding in the experimental cavity for the gamma measurement. The active detector is shielded by approximately 4 inches of lead provided by the commercial shield described in the text. Bottom: Cross section of GEANT4 geometry (side view) used in simulation of this setup. The active detector is shown in yellow. The only shielding present in this configuration is the commercial gamma shielding described in the text (the experimental cavity was otherwise empty).

reactor core gamma energy spectrum are washed out due to gammas interacting in the water, graphite, and lead shielding between the core and detector.

The different core positions were simulated in GEANT4, including a model of the lead shielding and the HPGe detector used to make the measurement (shown schematically in Figure 6). Each core position in simulation was systematically $2 \mathrm{~cm}$ further from the detector than the corresponding position in the data, due to a correction made to the measured position after the simulation had been run. For each core position, approximately $3 \times 10^{9}$ single gamma initial events were generated using the energy spectrum obtained from the MCNP core model described previously. The deposited energy of gammas reaching the detector were compared to the background-subtracted data, as shown in Figure 9. In this plot, the GEANT4 prediction was scaled to match the integrated event count of the data in the region of deposited energy greater than $100 \mathrm{keV}$. The energy resolution in the higher rate environment of the experimental cavity was dominated by pile-up effects resulting in a degradation of resolution with rate. A resolution smearing was applied to the simulated results to account for this effect, determined using a comparison of width of the $511 \mathrm{keV}$ line between the simulation and the data. A simple Gaussian fit was used to determine these line widths (ranging from about $4 \mathrm{keV}$ to $8 \mathrm{keV}$ ), and the difference of the squares of these widths was used to define a new Gaussian which was then applied as an event-by-event smearing to the simulated energy deposits. The simulation and data matched quite well in shape, with about a $25 \%$ deviation for the region above $3 \mathrm{MeV}$. The large deviation in the region below $20 \mathrm{keV}$ is due to detector threshold effects not accounted for in the simulation.

We determined the scaling of event rate as a function of core position in both the measured and the simulated spectra by taking the ratio of background-subtracted spectra at each position. This ratio is shown 

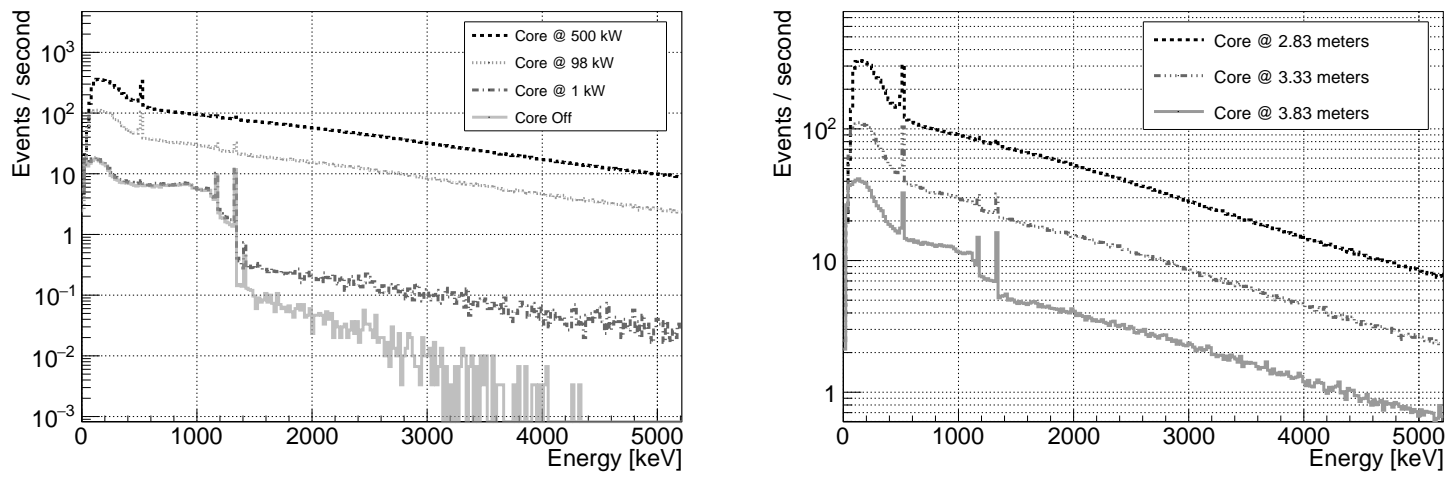

Figure 7: Measured gamma spectrum in the commercial HPGe detector (Canberra GC2020, approx. 0.5kg) for different reactor powers with the core held at 3.33 meters (left) and different reactor positions with the core at $98 \mathrm{~kW}$ power (right). All runs consist of 300 seconds of live time.
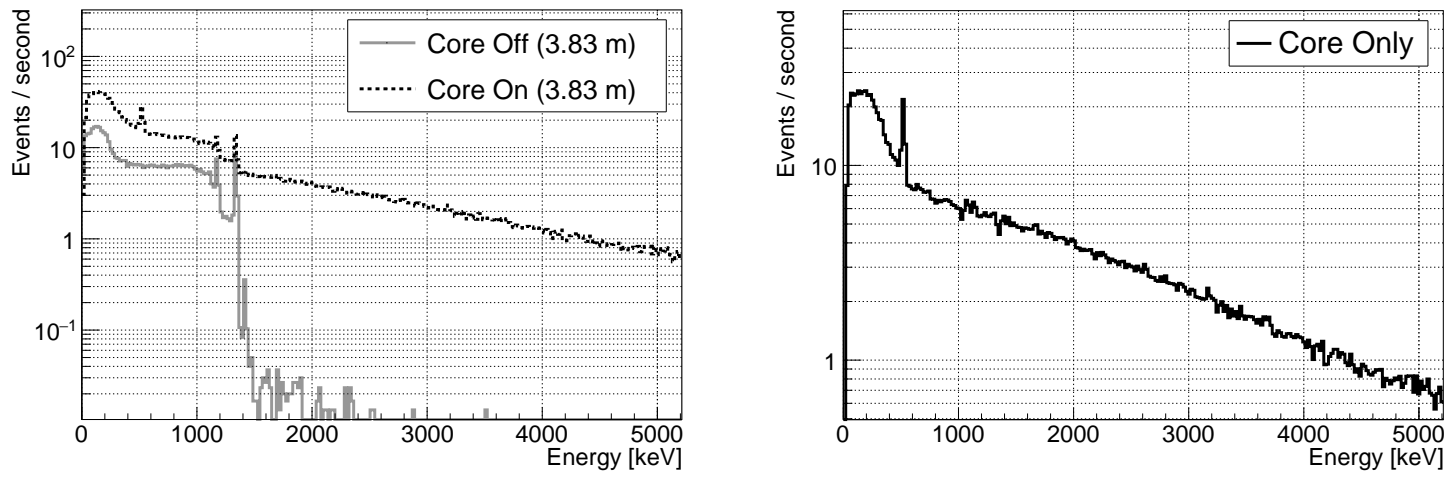

Figure 8: Full measured gamma spectrum in HPGe detector with the core (at distance $3.83 \mathrm{~m}$ ) on and with the core off (left). The core-off spectrum is subtracted from the core-on spectrum to get the "core-only" spectrum (right) which can be compared directly to the simulation. The main features of the core-off spectrum are the two Cobalt 60 gamma lines at 1173.2 and 1332.5 $\mathrm{keV}$ which come from activated stainless steel lining near the back of the experimental cavity.

in Figure 10. The rate is reduced by roughly a factor of 3.5 per $0.5 \mathrm{~m}$ of increased distance from the core in both the data and the simulation. Because this scaling of rate with distance is reproduced to within about $10-15 \%$ in the simulation, the scaling factor needed to translate the simulated result to a rate is taken to be constant regardless of the distance of the core to the detector. We take as this scaling factor the ratio of the integrated event count of the data (in the region of deposited energy greater than $100 \mathrm{keV}$ ) to the integrated weighted event count in the same region in the simulated result. This scaling factor was applied to all further simulated results to obtain rate estimations.

\section{Neutron Background Measurements}

Due to administrative and safety constraints preventing deployment of traditional detectors, the background neutron measurement was thus far restricted to measurements needed for validation of the computational models. The validation measurement was performed using a $6 \times 6$ inch copper foil (see Figure 11) that was activated by neutrons in the experimental cavity. The copper acts as an absorber of thermal neutrons and can be used to verify the integrated thermal neutron flux by measuring the activation of the foil after neutron exposure. This measurement was performed with the core and experimental cavity in the configuration shown in Figure1 (left) (i.e., without any additional shielding). 


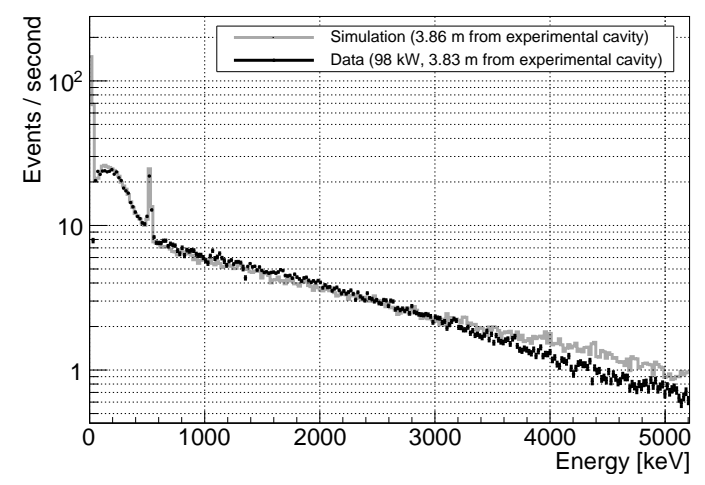

Figure 9: Comparison of reactor core gamma spectrum measured by the Canberra HPGe detector and the prediction from the GEANT4 simulation. The GEANT4 prediction is scaled to match the integrated event count of the data for the region of deposited energy greater than $100 \mathrm{keV}$ in order to compare shape. The large deviation in the region below $20 \mathrm{keV}$ is due to detector threshold effects not accounted for in the simulation.

Using the MCNP simulation, the neutron spectrum at the surface of the graphite box facing the experimental cavity was determined using the 172 energy bin XMAS structure 12. The calculated spectrum is shown in Figure 11 (right), with fluxes of 5.6(3) $\times 10^{6} \mathrm{~cm}^{-2} \mathrm{~s}^{-1}$ fast and $4.0(2) \times 10^{10} \mathrm{~cm}^{-2} \mathrm{~s}^{-1}$ thermal neutrons, and was used in a subsequent activation analysis to obtain the capture cross-section of ${ }^{63} \mathrm{Cu}$. This cross-section was then used in the Bateman equations 13 to obtain the total thermal flux from the ${ }^{64} \mathrm{Cu}$ measured activity. The activated ${ }^{64} \mathrm{Cu}$ decays with a half life of 12.7 hours via electron capture, beta and positron emission $(0.5787 \mathrm{MeV}$ and $0.6531 \mathrm{MeV}$ respectively), and gamma emission $(1.355 \mathrm{MeV})$. The activity of the foil was measured by a HPGe detector at the NSC, resulting in a measured total thermal neutron flux of $5.8(3) \times 10^{7} \mathrm{~cm}^{-2} \mathrm{~s}^{-1}$. The uncertainty on these fluxes include both statistical uncertainty from the model calculation and HPGe activation measurement as well as uncertainty on the core power calibration.

The measured thermal neutron flux together with a calculated thermal neutron flux profile is shown in Figure 12. The data point was consistent with the expected result from the simulation within $5 \%$, indicating that the neutron flux is predicted by the MCNP core model.

\section{Muon Background Measurements}

Bolometric detectors with low thresholds are particularly vulnerable to large energy depositions from atmospheric muons. A typical solution for low rate experiments to this problem is to install such detectors deep underground, maximizing the overburden, and thus shielding of the detector. For detecting higher rate processes, such as neutrino interactions near a nuclear reactor, a higher muon rate can be tolerated. The experimental cavity proposed for this experiment provides some overburden in the form of the high density concrete wall surrounding the reactor pool, as the cavity is located within this wall (see Figure 1). This overburden has been characterized with regards to its muon shielding effectiveness by measurements described below.

Two polyvinyl-toluene scintillators (one smaller $1.75 \times 0.5 \times 0.375 \mathrm{in}^{3}$ panel on top and a larger $13.0 \times 3.0 \times$ $0.375 \mathrm{in}^{3}$ panel on bottom, separated by a 2 in lead brick) were installed to trigger on muons. These counters were coupled to photomultiplier tubes via waveguides and were connected to front-end NIM electronics to produce a coincidence signal when both scintillators triggered above threshold within a $3 \mathrm{~ns}$ window of each other. Due to the high rate (and high energy) gamma environment, accidental triggers due to random coincidence were an experimental concern. To characterize the rate of such events, the signal of one scintillator was delayed arbitrarily to about $150 \mathrm{~ns}$, maintaining all other aspects of the experimental conditions. This setup was first exposed in the most radioactive location surveyed to get an upper limit on the rate of random coincidence events. A $13 \mathrm{hr}$ run under these conditions showed no events passing the 

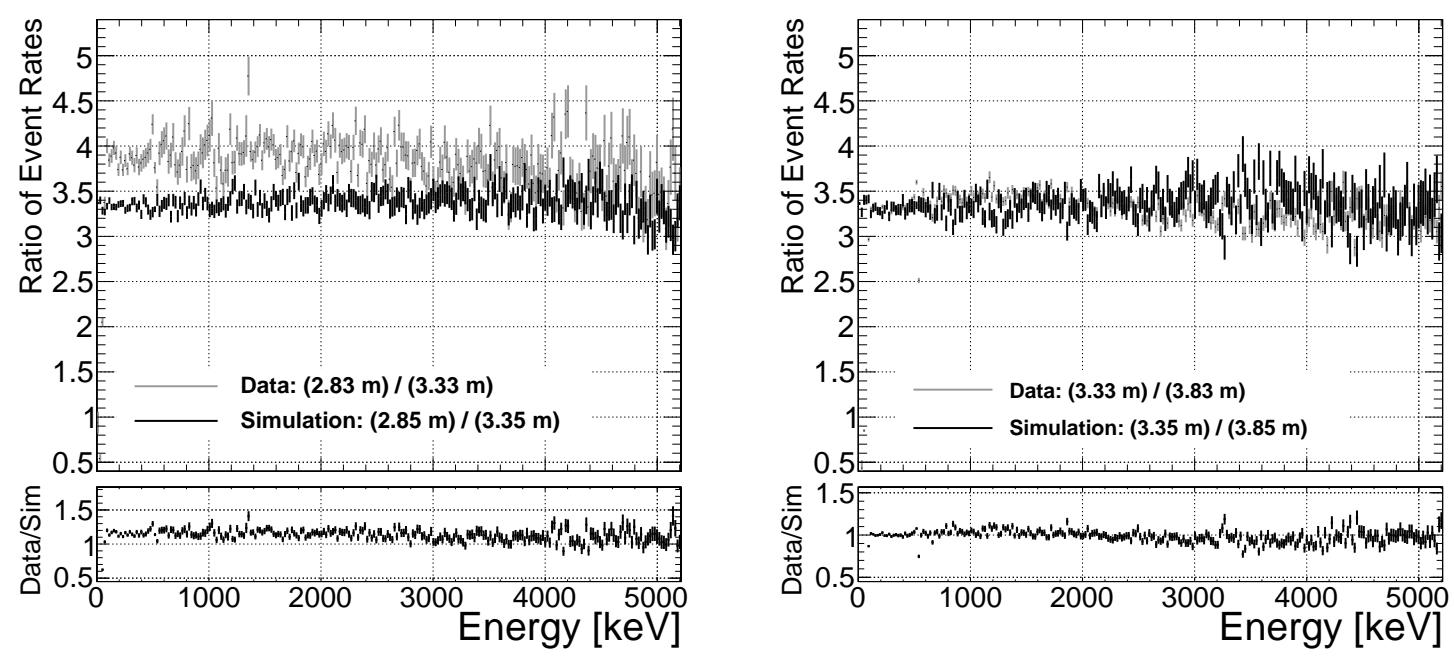

Figure 10: Event rate scaling as a function of deposited energy in the HPGe detector for different reactor core positions in the measured data and simulation. Error bars are statistical uncertainties only.

coincidence requirement, demonstrating that a subtraction correction for random coincidence would not be necessary.

Muon measurements were made with this setup at 5 locations. The first was made in a building adjacent to the reactor confinement building as a baseline measurement. This location has no effective overburden and was used as a open-sky muon baseline reference. With this setup, a rate of about $1 \mu / \mathrm{min} / \mathrm{cm}^{2}$ was measured at that location. The equipment was then moved to the lower research level of the reactor confinement building. The other measurements were made in the confinement building and all share the overburden of the $1 \mathrm{~m}$ thick high density concrete roof. The next measurement, performed outside of the reactor pool wall, showed a $17 \%$ reduction in muon rate compared to the baseline. The setup was then installed in the experimental cavity, inserted into 3 different positions in the cavity as shown in Figure 13 The measured reduction in muon rate with respect to the open-sky baseline is given in Table 1.

\begin{tabular}{ccc} 
Position \# & Distance Into Cavity & Muon Rate Reduction \\
\hline 1 & - & $17 \pm 6 \%$ \\
2 & $1.0 \mathrm{~m}$ & $50 \pm 3 \%$ \\
3 & $1.5 \mathrm{~m}$ & $57 \pm 6 \%$ \\
4 & $2.5 \mathrm{~m}$ & $47 \pm 4 \%$
\end{tabular}

Table 1: The muon rate reduction with respect to the open-sky measurement. Position number refers to the positions marked on the diagram in Figure 13 The open-sky measurement was taken in a separate building at the NSC facility located at ground level.

These measurements show a $50 \%$ reduction of muons incident upon the MINER detectors in the proposed experimental cavity. They also guide calculations of energy depositions that will be carefully considered in finalizing the geometry of the detectors. For a given volume/mass, one must make a trade-off between muon rate (determined effectively by a detector's horizontal cross-section) and energy deposition per muon (determined by detector's vertical dimension).

\section{Rate Estimate With Shielding}

We then used the GEANT4 setup to estimate the backgrounds with a full preliminary shielding design, as shown in Figure 5. We generated approximately $3 \times 10^{9}$ gamma and $4 \times 10^{9}$ neutron events with the 

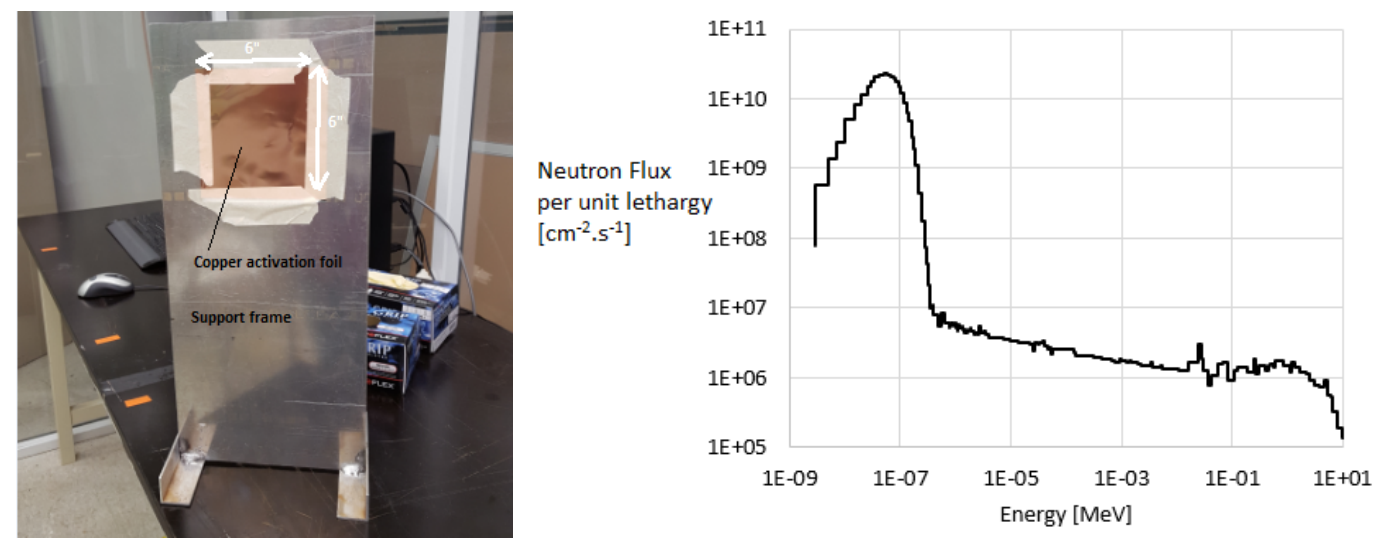

Figure 11: Photo of the $6 \times 6$ in copper foil setup which was placed in the experimental cavity to be irradiated by neutrons(left). Neutron spectrum inside the experimental cavity at the surface of the graphite block facing the experimental cavity, as calculated by MCNP (right). The neutron spectrum is bin-by-bin normalized to unit lethargy, with normalization factor given as $E_{\text {Ave }} /\left(E_{2}-E_{1}\right)$ where $E_{1}$ and $E_{2}$ are the energy values at the respective bin edges and $E_{A v e}$ is the average of these bin edge values.

core at the closest possible proximity to the experimental cavity (at the face of the graphite block shown in Figure 1). The simulation included 4 germanium detectors and 4 silicon detectors, each represented as $100 \mathrm{~mm}$ diameter, $33 \mathrm{~mm}$ thickness cylinders, and backgrounds were assessed by determining the energy deposited in these volumes. Rates were determined using the scaling obtained in the previous gamma and neutron measurements. The resulting spectrum of energy deposited is shown in Figure 14.

These rates are compatible with the target background rate of 100 events $/ \mathrm{kg} /$ day in the range of recoil energy between 10 and $1000 \mathrm{eV}_{\mathrm{nr}}$ and optimization of the shielding configuration will further reduce the estimated rate. The event rate outside of this window was approximately $30 \mathrm{~Hz}$, found by converting events/day to events/sec and multiplying by the mass of the $4 \mathrm{Ge}$ and $4 \mathrm{Si}$ detectors used in this simulation. This total event rate is below the acceptable upper limit of about $100 \mathrm{~Hz}$ set by our data acquisition hardware. It should be noted that moving the core further away would drastically reduce the expected background, due to the addition of more water shielding between core and experiment, as well as the $r^{-2}$ reduction with distance. This estimate gives us confidence that we are within reach of our background goal with a reasonable shielding configuration and within a core to experiment distance compatible with making our signal measurement.

\section{Summary and Future Prospects}

We have performed in situ measurements and detailed simulations of expected backgrounds for the proposed MINER experiment with the goal of detecting CE $\nu$ NS. Simulations reproduce the measurements of thermal neutrons and gammas and were used to estimate the expected backgrounds with a full shielding designed to bring the backgrounds down to a level compatible with a measurement of the CE $\nu \mathrm{NS}$ signal in the MINER experiment. This simulation has shown that it is indeed possible to reduce both neutron and gamma backgrounds to a level of about 100 events $/ \mathrm{kg} /$ day in the range of recoil energy between 10 and $1000 \mathrm{eV}_{\mathrm{nr}}$ with the reactor core as close as about $2.3 \mathrm{~m}$.

Gamma measurements will be performed with increased lead shielding and a core at the closest position to the experiment. Neutron measurements will be performed with other foil samples to provide multiple checks against the simulation, as well as with other detector technologies. In parallel, optimization of the shielding configuration is being performed in both the GEANT4 and MCNP simulations to further reduce the neutron and gamma background. Simulation of muon-induced neutron backgrounds are now underway, but these are expected to be small in the experimental region of interest. 


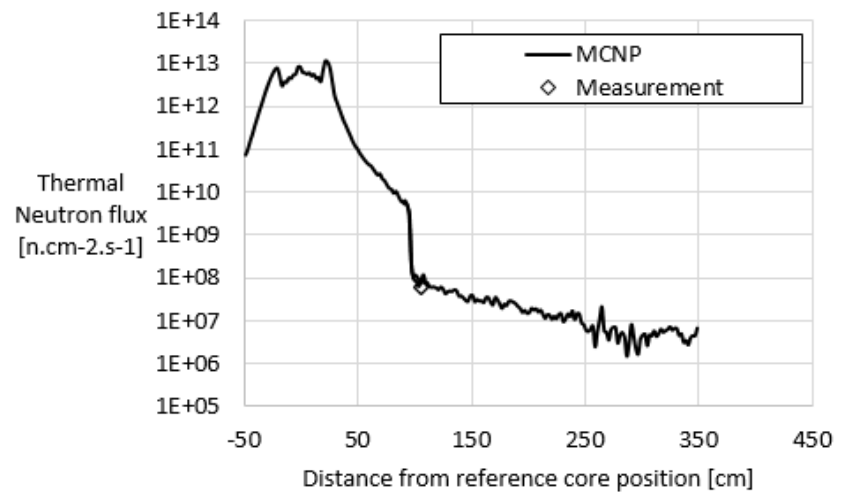

Figure 12: Thermal neutron flux as a function of distance from the reactor core center with only the graphite block between core and foil position. The data point represents the flux calculated using the measured activation of the copper foil at that position. The solid line indicates the flux calculated directly from the MCNP core model.

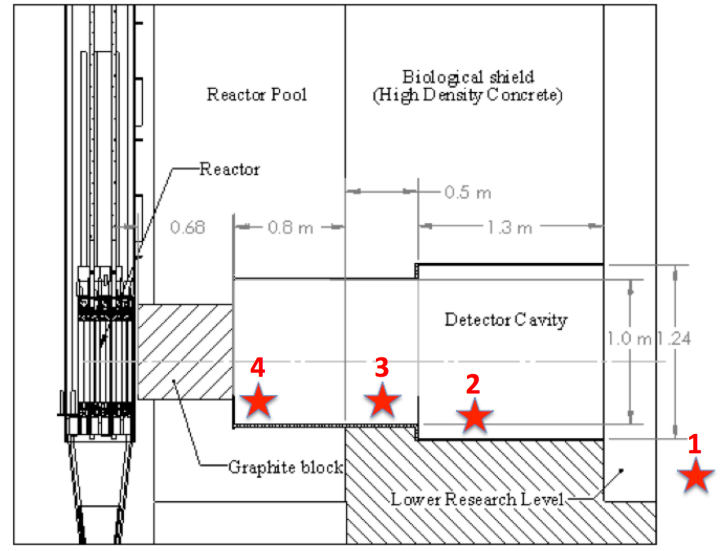

Figure 13: Approximate position of the muon scintillation detector in the experimental cavity for the four measurements made in or near the experimental cavity. A baseline measurement not shown in this figure was made in an adjacent building with minimal overburden.

Based on measurements reported here and planned in the future, we are developing plans for in situ monitoring of backgrounds for the MINER experiment. Several technologies are being considered including segmented, active liquid-scintillator shield, and a dedicated iZIP-type detector used by SuperCDMS [14] that provides excellent nuclear recoil discrimination down to $1 \mathrm{keV}$ recoil energy. Also, to further monitor and characterize neutron backgrounds, a ${ }^{6} \mathrm{Li}$ doped scintillator detector for thermal neutrons, and a PTP-doped scintillator with neutron/gamma pulse shape discrimination for fast neutrons are being constructed at the Texas A\&M University Cyclotron Institute.

\section{Acknowledgements}

The authors gratefully acknowledge the Mitchell Institute for Fundamental Physics and Astronomy for seed funding, as well as the Brazos HPC cluster at Texas A\&M University (brazos.tamu.edu) and the Texas Advanced Computing Center (TACC) at the University of Texas at Austin (www.tacc.utexas.edu) for providing resources that have contributed to the research results reported within this paper. We also gratefully 


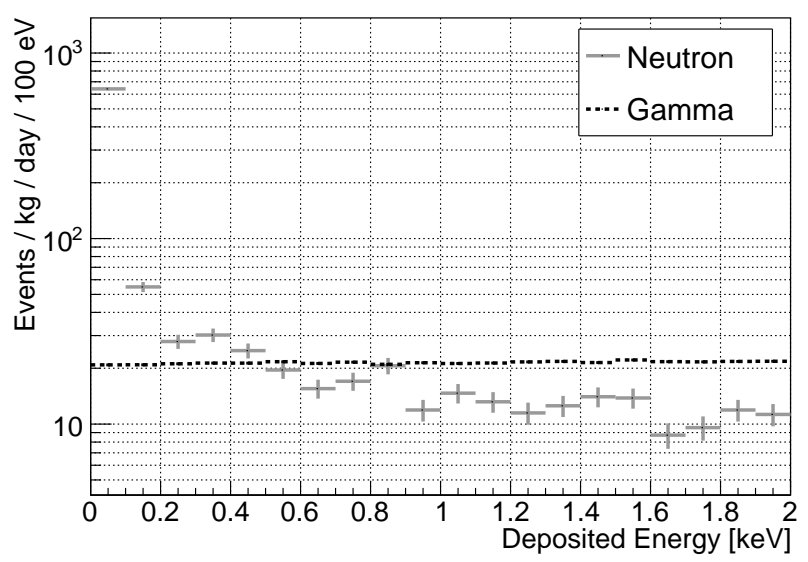

Figure 14: Energy deposited in the germanium detectors due to neutron and gamma backgrounds as estimated using the GEANT4 simulation and shielding design shown in Figure 5 The core source was located at the closest possible proximity to the experimental cavity. Uncertainties shown on this plot are statistical only.

acknowledge the TAMU Nuclear Science Center for facilitating the MINER experiment and providing numerous resources and advice in the course of planning and developing this program. R.M, R.H. and N.M acknowledge the support of DOE grant DE-SC0014036 in development of synergistic activities with the SuperCDMS collaboration. G.V.R. acknowledges support by the U.S. Department of Energy, Office of Science, Office of Nuclear Science, under Award No. DE-FG02-93ER40773 and also support by the Welch Foundation (Grant No. A-1853). J.W.W. acknowledges support from NSF grant PHY-1521105 and the Mitchell Institute for Fundamental Physics and Astronomy. L.S. acknowledges support from NSF grant PHY-1522717. B.D. acknowledges support from DOE grant DE-FG02-13ER42020.

\section{References}

[1] D. Z. Freedman, "Coherent neutrino nucleus scattering as a probe of the weak neutral current," Phys. Rev., vol. D9, pp. 1389-1392, 1974.

[2] N. Mirabolfathi, H. R. Harris, R. Mahapatra, K. Sundqvist, A. Jastram, B. Serfass, D. Faiez, and B. Sadoulet, "Toward Single Electron Resolution Phonon Mediated Ionization Detectors," 2015.

[3] P. N. Luke, J. Beeman, F. S. Goulding, S. E. Labov, and E. H. Silver, "Calorimetric ionization detector," Nucl. Instrum. Meth., vol. A289, pp. 406-409, 1990.

[4] J. Barranco, O. G. Miranda, and T. I. Rashba, "Probing new physics with coherent neutrino scattering off nuclei," JHEP, vol. 12 , p. $021,2005$.

[5] K. Scholberg, "Prospects for measuring coherent neutrino-nucleus elastic scattering at a stopped-pion neutrino source," Phys. Rev., vol. D73, p. 033005, 2006.

[6] B. Dutta, Y. Gao, A. Kubik, R. Mahapatra, N. Mirabolfathi, L. E. Strigari and J. W. Walker, Phys. Rev. D 94, no. 9, 093002 (2016) doi:10.1103/PhysRevD.94.093002 [arXiv:1511.02834 [hep-ph]].

[7] B. Dutta, R. Mahapatra, L. E. Strigari, and J. W. Walker, "Sensitivity to $Z$-prime and nonstandard neutrino interactions from ultralow threshold neutrino-nucleus coherent scattering," Phys. Rev., vol. D93, no. 1, p. 013015, 2016.

[8] J. B. Dent, B. Dutta, S. Liao, J. L. Newstead, L. E. Strigari and J. W. Walker, arXiv:1612.06350 [hep-ph].

[9] T. Goorley et al., "Initial MCNP6 Release Overview," Nucl. Tech., vol. 180, pp. 298-315, 2012.

[10] S. Agostinelli et al., "GEANT4: A Simulation toolkit," Nucl. Instrum. Meth., vol. A506, pp. 250-303, 2003.

[11] "Shielding physics list description." http://www.slac.stanford.edu/comp/physics/geant4/slac_physics_lists/ shielding/physlistdoc.html, 2016 (accessed Feb, 2016).

[12] D. Cacuci, Handbook of Nuclear Engineering Vol 1: Nuclear Engineering Fundamentals. Springer US, 2010. ISBN:9780-387-98149-9.

[13] H. Bateman, "Solution of a system of differential equations occurring in the theory of radioactive transformations," Proc. Cambridge Phil. Soc., vol. 15, pp. 423-427, 1910.

[14] P. L. Brink et al., "First test runs of a dark-matter detector with interleaved ionization electrodes and phonon sensors for surface-event rejection," Nucl. Instrum. Meth., vol. A559, pp. 414-416, 2006. 